\title{
A Comparative Study of Sea Clutter Covariance Matrix Estimators
}

\author{
Ding Tao, Stian Normann Anfinsen, Member, IEEE, and Camilla Brekke, Member, IEEE
}

\begin{abstract}
Estimation of the polarimetric covariance matrix is an important task in statistical modeling of sea clutter for maritime applications of polarimetric synthetic aperture radar (PolSAR) data. This work provides a comprehensive study of four covariance matrix estimators: the maximum likelihood estimators under the Gaussian distribution (G-ML) and the K distribution (K-ML), an approximation of the latter (AK-ML), and a robust M-estimator. It adds to previous theoretical studies of these algorithms by evaluating their performance with respect to both estimation accuracy and computational efficiency. Experiments are performed on simulated datasets. Various texture conditions of the sea clutter are considered in the study.
\end{abstract}

Index Terms - Synthetic aperture radar, Polarimetry, Sea clutter, Statistical modeling, Covariance matrix estimation, Texture.

\section{INTRODUCTION}

$\mathbf{P}$ OLARIMETRIC synthetic aperture radar (PolSAR) produces high quality measurements of the Earth surface, and has been widely utilized in different remote sensing applications. Sea clutter is an example of a natural surface which consists of distributed scatterers, and where all polarimetric information is contained in the covariance matrix of the single-look complex (SLC) scattering vector. Therefore, covariance matrix estimation is an important factor in the analysis of PolSAR images of the sea. For instance, constant false alarm rate (CFAR) ship detection based on PolSAR imagery relies upon accurate statistical estimation of the sea clutter [1]-[3]. The covariance matrix estimate is also applied in the polarimetric whitening filter (PWF), which processes the SLC scattering vector into a full-resolution pixel intensity and provides effective speckle reduction [4].

In this study, the main objective is to investigate and compare different covariance matrix estimators for sea clutter. The covariance matrix is commonly estimated from a neighborhood of pixels within a sliding window. The classical sample covariance matrix (SCM) estimator has been applied as a good default option in previous studies. It is the maximum likelihood (ML) covariance matrix estimator under the simple assumption that the distribution of the scattering vector is

Manuscript received April 15, 2013; revised June 28 and October 1, 2013; accepted October 1, 2013. Date of publication XXX XX, XXXX; date of current version $\mathrm{XXX} \mathrm{XX}, \mathrm{XXXX}$.

Ding Tao, Stian Normann Anfinsen, and Camilla Brekke are with the Department of Physics and Technology, UiT The Arctic University of Norway, 9037 Troms $\emptyset$, Norway (e-mail: tao.ding@uit.no; stian.normann.anfinsen@uit.no; camilla.brekke@uit.no).

Color versions of one or more of the figures in this paper are available online at http://ieeexplore.ieee.org.

Digital Object Identifier xx.xxxx/LGRS.xxxx.xxxxxxx complex, circular and zero-mean multivariate Gaussian. The ML estimators both for the general case of a compound Gaussian scattering vector and the particular case of gamma distributed texture were derived in [5]. An approximation for this case is introduced here, which provides comparable accuracy and reduces the computational cost. The M-estimator for the covariance matrix was introduced in [6] as an efficient alternative to the ML estimator, and has recently won popularity in the PolSAR literature [7]-[9], where it is known as the fixed-point (FP) estimator.

Whereas the theoretical properties of the ML estimator and the M-estimator have been studied in detail [7], [8], little has been done to assess and compare estimators for the sea clutter covariance matrix in terms of accuracy and speed. This work provides a systematic study of the emerging candidates and examines their performance under various texture conditions, ranging from low through moderate to high cases. In the experiments, simulated sea clutter is used as reference data in a quantitative accuracy assessment. However, the model covariance matrix used to generate data is obtained from a real Radarsat-2 quadrature polarimetric (quad-pol) dataset.

This paper is organized as follows. Section II gives a brief introduction to sea clutter statistics. Section III is devoted to a systematic outline of covariance matrix estimation approaches and detailed descriptions of each estimator. In Section IV, different covariance matrix estimators are examined under various texture conditions. Their performance is evaluated in terms of estimation accuracy and computational efficiency. Section V presents the main conclusions.

\section{Sea Clutter statistics}

The PolSAR image provides a representation of the local scattering properties of the sea clutter. The scattering vector, i.e., the Sinclair matrix represented in vector form, is the basis for statistical data analysis and modeling. It is defined as

$$
s=\left[\begin{array}{llll}
s_{h h} & s_{h v} & s_{v h} & s_{v v}
\end{array}\right]^{\prime},
$$

where $[\cdot]^{\prime}$ is the transposition operator and $s_{t r}$ represents complex scattering coefficients subscripted with transmit polarization $t$ and receive polarization $r$, that can be horizontally $(h)$ or vertically $(v)$ oriented. The vector samples $\left\{\boldsymbol{s}_{i}\right\}_{i=1}^{n}$ of size $n$ obtained in local neighborhoods of the sea are assumed independently and identically distributed (IID). Eq. (1) shows the quad-pol SLC measurement of a SAR system.

The product model is commonly assumed to be an appropriate statistical model for the sea clutter in various scenarios. 
Introduced by Yueh et al. [10], the multivariate product model decomposes the scattering vector as

$$
s=\sqrt{\tau} \boldsymbol{x},
$$

where the real and positive random texture variable, $\tau \in \mathbb{R}^{+}$, represents spatial variability of the reflectivity, and the speckle is represented by an independent $d$-dimensional circular complex Gaussian vector, $\boldsymbol{x} \sim \mathcal{N}_{d}^{\mathbb{C}}(0, \Sigma)$, with zero mean vector and covariance matrix $\Sigma$. A study by Vachon et al. [2] indicates that sea clutter imaged by the Envisat ASAR Cband sensor can be significantly non-Gaussian. Therefore, it seems appropriate to assume e.g. a gamma distributed texture variable, in which case the scattering vector will be multivariate $\mathrm{K}$ distributed [10].

When a gamma distributed texture variable is assumed, it introduces an additional shape parameter, $\alpha$, which must be determined to define the distribution. The shape parameter indicates the texture condition; the larger (smaller) the shape parameter, the lower (higher) the texture variation level. The Gaussian case with no texture corresponds to $\alpha=\infty$. Moment-based methods for shape parameter estimation have been studied in [11], [12]. Experiments verify that the variance of moment-based estimator increases with moment order. In this study, we use a method based on fractional moments of single-look intensity, which is applied to all available polarimetric channels before averaging the results, as in [13]. From [14], the $m$ th-order moment of a K-distributed SAR intensity $I$ is given as

$$
E\left\{I^{m}\right\}=E\{I\}^{m} \frac{\Gamma(L+m)}{L^{m} \Gamma(L)} \frac{\Gamma(\alpha+m)}{\alpha^{m} \Gamma(\alpha)},
$$

where $E\{\cdot\}$ is the expectation operator, $L$ is the number of looks, $\Gamma(\cdot)$ is the gamma function, and $\alpha$ is the shape parameter. In the method of fractional moments, the moment order is set to $m=0.5$, and eq. (3) is solved numerically for $\alpha$ after the population moments have been replaced by sample moments computed from data. The sample moments may attain values for which eq. (3) has no solution. This happens when the sample moments fall outside the admissible region for the population moments. It has no consequence in practice, since this case can be interpreted as $\hat{\alpha}=\infty$, and we revert to a Gaussian model for the scattering vector.

\section{COVARIANCE MATRIX ESTIMATORS}

To obtain a nonsingular estimate of the covariance matrix, we need a data sample of size $n \geq d$ from the PolSAR imagery. The covariance matrix estimate for a given pixel is usually based on a neighborhood of pixels, and a sliding window technique is used to produce an estimate for the whole image. In general, the covariance matrix estimator is formulated as

$$
\hat{\boldsymbol{C}}=\frac{1}{n} \sum_{k=1}^{n} w \cdot \boldsymbol{s}_{k} \boldsymbol{s}_{k}^{\dagger}
$$

where $n$ is the number of samples used for estimation, $\dagger$ is the complex conjugate transpose operator, and $w$ is a weighting function distinguishing different estimation approaches.

\section{A. Maximum likelihood estimator}

In [5], Gini and Greco derived the general ML covariance matrix estimator based on the product model, which is given as

$$
\hat{\boldsymbol{C}}_{M L}^{(i+1)}=\frac{1}{n} \sum_{k=1}^{n} \frac{h_{d+1}\left(\boldsymbol{s}_{k}^{\dagger}\left(\hat{\boldsymbol{C}}_{M L}^{(i)}\right)^{-1} \boldsymbol{s}_{k}\right)}{h_{d}\left(\boldsymbol{s}_{k}^{\dagger}\left(\hat{\boldsymbol{C}}_{M L}^{(i)}\right)^{-1} \boldsymbol{s}_{k}\right)} \cdot \boldsymbol{s}_{k} \boldsymbol{s}_{k}^{\dagger},
$$

where $i=0,1,2, \ldots, N$ is the iteration number and $N$ is the number of iterations, $d$ is the dimension of the scattering vector, and the function $h_{d}(q)$ is defined as

$$
h_{d}(q)=\int_{0}^{+\infty} \tau^{-d} \exp \left(-\frac{q}{\tau}\right) p_{\tau}(\tau) d \tau,
$$

with the probability density function (pdf) of texture, $p_{\tau}(\tau)$. Eq. (5) is an iterative solution of a transcendental equation. The estimate at the $(i+1)$ th step is obtained from the estimate at step $i$. This process requires prior knowledge of the texture pdf $p_{\tau}(\tau)$ and an appropriate starting point.

Eq. (5) can be rewritten by defining the weighting function $w_{o}(q)=h_{d+1}(q) / h_{d}(q)$. Then the general ML covariance matrix estimator is written as

$$
\hat{\boldsymbol{C}}_{M L}^{(i+1)}=\frac{1}{n} \sum_{k=1}^{n} w_{o}\left(\boldsymbol{s}_{k}^{\dagger}\left(\hat{\boldsymbol{C}}_{M L}^{(i)}\right)^{-1} \boldsymbol{s}_{k}\right) \cdot \boldsymbol{s}_{k} \boldsymbol{s}_{k}^{\dagger} .
$$

To implement it, a texture pdf must be assumed.

1) Gaussian distribution based ML estimator: The simplest special case of the product model is when we assume a constant texture variable. The scattering vector then becomes complex multivariate Gaussian distributed, and the ML estimator reduces to the familiar SCM estimator whose weighting function is constant, $w_{G}=1$. It can thus be written as

$$
\hat{\boldsymbol{C}}_{G}=\frac{1}{n} \sum_{k=1}^{n} 1 \cdot \boldsymbol{s}_{k} \boldsymbol{s}_{k}^{\dagger} .
$$

This approach is the computationally most simple, but unfortunately, it suffers a performance loss in spiky clutter [1]. Note that the Gaussian distribution based ML (G-ML) estimate can be used to initialize all of the iterative estimators and is known to be complex Wishart distributed [15].

2) $K$ distribution based $M L$ estimator: For real sea clutter, the constant texture assumption is often too simple and inaccurate. In this study, gamma distributed texture with unit mean value $(E\{\tau\}=1)$ is assumed, thus the scattering vector becomes multivariate $\mathrm{K}$ distributed [10]. The pdf of the mean normalized gamma distributed texture variable $\tau$ is defined as

$$
p_{\tau}(\tau)=\frac{1}{\Gamma(\alpha)}(\alpha)^{\alpha} \tau^{\alpha-1} \exp (-\alpha \tau),
$$

where $\alpha$ is the shape parameter. The weighting function for this case was found in [5] as

$$
w_{K}(q)=\sqrt{\frac{\alpha}{q}} \frac{K_{\alpha-d-1}(\sqrt{4 \alpha q})}{K_{\alpha-d}(\sqrt{4 \alpha q})},
$$

where $K_{\nu}(q)$ is the modified Bessel function of the second kind with order $\nu$. Therefore, the $\mathrm{K}$ distribution based ML (K-ML) estimator becomes

$$
\hat{\boldsymbol{C}}_{K}^{(i+1)}=\frac{1}{n} \sum_{k=1}^{n} w_{K}\left(\boldsymbol{s}_{k}^{\dagger}\left(\hat{\boldsymbol{C}}_{K}^{(i)}\right)^{-1} \boldsymbol{s}_{k}\right) \cdot \boldsymbol{s}_{k} \boldsymbol{s}_{k}^{\dagger} .
$$


This transcendental equation still needs to be solved iteratively, which includes evaluating the complicated Bessel $\mathrm{K}$ function. Note that the shape parameter of the texture pdf must be estimated. Hence, we have a compound estimation problem.

3) Approximate $K$ distribution based ML estimator: Approximations of the K-ML estimator can be achieved through a number of methods. The authors have recently proposed one possible option [3]. It computes the approximate bounds of the Bessel $\mathrm{K}$ function ratio in eq. (10), but suffers serious limitation for highly textured clutter. An interesting interpretation of $w_{o}$ and some intuitive approximations of $w_{K}$ are given in [16] in the context of polarimetric target detection and estimation theory.

To obtain the required accuracy, a new approximate $\mathrm{K}$ distribution based ML (AK-ML) estimator is derived by approximating the nonlinear memoryless function $h_{d}(q)$ defined in eq. (6). After inserting the unit mean gamma distribution in eq. (9) as the texture distribution, it is rewritten as

$$
h_{d}(q)=\frac{\alpha^{\alpha}}{\Gamma(\alpha)} \int_{0}^{\infty} \exp (f(\tau ; \alpha, q)) d \tau
$$

by means of the function

$$
f(\tau ; \alpha, q)=(\alpha-d-1) \ln \tau-\frac{q}{\tau}-\alpha \tau .
$$

In order to use Laplace's method to approximate the integral in eq. (12), we find the first and second derivative of $f(\tau)$ as

$$
\begin{aligned}
f^{\prime}(\tau ; \alpha, q) & =\frac{(\alpha-d-1)}{\tau}+\frac{q}{\tau^{2}}-\alpha, \\
f^{\prime \prime}(\tau ; \alpha, q) & =-\frac{(\alpha-d-1)}{\tau^{2}}-\frac{2 q}{\tau^{3}} .
\end{aligned}
$$

Note that the shape parameter $\alpha$ is strictly positive, as is the texture variable $\tau$. As seen from eq. (11), the input to $h_{d}(q)$ is a quadratic form in a positive definite matrix, so $q$ is also positive. Extrema of $f(\tau)$ are located at solutions of $f^{\prime}(\tau)=0$, which is equivalent to $\alpha \tau^{2}-(\alpha-d-1) \tau-q=0$. The only positive and thus physically valid solution is

$$
\tau_{0}=\frac{\beta+\sqrt{\beta^{2}+4 \alpha q}}{2 \alpha},
$$

where $\beta=\alpha-d-1$. The second derivative at $\tau_{0}$ is $f^{\prime \prime}\left(\tau_{0}\right)=$ $-\left(\beta \tau_{0}+2 q\right) / \tau_{0}^{3}$. By showing

$$
\beta \tau_{0}=\frac{\beta^{2}}{2 \alpha}\left(1+\sqrt{1+\frac{4 \alpha q}{\beta^{2}}}\right)>0,
$$

it follows that $f^{\prime \prime}\left(\tau_{0}\right)<0$, and we can conclude that $f\left(\tau_{0}\right)=$ $\beta \ln \tau_{0}-q / \tau_{0}-\alpha \tau_{0}$ is the unique maximum of $f(\tau)$. The Laplace approximation can then be applied. It yields [17]

$$
\begin{aligned}
& h_{d}(q) \simeq \frac{\alpha^{\alpha}}{\Gamma(\alpha)}\left(\frac{2 \pi}{-f^{\prime \prime}\left(\tau_{0}\right)}\right)^{\frac{1}{2}} e^{f\left(\tau_{0}\right)} \\
& =\frac{\alpha^{\alpha}}{\Gamma(\alpha)}\left(\frac{2 \pi \tau_{0_{d}}^{3}}{\beta_{d} \tau_{0_{d}}+2 q}\right)^{\frac{1}{2}} \tau_{0_{d}}^{\beta_{d}} \exp \left(-\frac{q}{\tau_{0_{d}}}-\alpha \tau_{0_{d}}\right),
\end{aligned}
$$

where the indices of $\beta_{d}$ and $\tau_{0_{d}}$ indicate that they depend on $d$. Hence, the weighting function of the K-ML estimator $h_{d+1}(q) / h_{d}(q)$ is approximated by

$$
\begin{gathered}
w_{A K}(q)=\left(\frac{\beta_{d}+\frac{2 q}{\tau_{0_{d}}}}{\beta_{d+1}+\frac{2 q}{\tau_{0_{d+1}}}}\right)^{\frac{1}{2}} \frac{\tau_{0_{d+1}}^{1+\beta_{d+1}}}{\tau_{0_{d}}^{1+\beta_{d}}} \\
\times \exp \left(\left(\frac{q}{\tau_{0_{d}}}-\frac{q}{\tau_{0_{d+1}}}\right)+\alpha\left(\tau_{0_{d}}-\tau_{0_{d+1}}\right)\right),
\end{gathered}
$$

with the previously given expressions for $\beta$ and $\tau_{0}$ inserted.

\section{B. M-estimator}

A rather different approach was proposed by Tyler [6], who derived his covariance matrix estimator as a particular case of Huber's general class of robust ML-type estimators, known as M-estimators. The M-estimator is defined as

$$
\begin{aligned}
\hat{\boldsymbol{C}}_{M}^{(i+1)} & =\frac{1}{n} \sum_{k=1}^{n} \frac{d}{\boldsymbol{s}_{k}^{\dagger}\left(\hat{\boldsymbol{C}}_{M}^{(i)}\right)^{-1} \boldsymbol{s}_{k}} \cdot \boldsymbol{s}_{k} \boldsymbol{s}_{k}^{\dagger} \\
& =\frac{1}{n} \sum_{k=1}^{n} w_{M}\left(\boldsymbol{s}_{k}^{\dagger}\left(\hat{\boldsymbol{C}}_{M}^{(i)}\right)^{-1} \boldsymbol{s}_{k}\right) \cdot \boldsymbol{s}_{k} \boldsymbol{s}_{k}^{\dagger},
\end{aligned}
$$

where $w_{M}(q)=d / q$. Most notably, the M-estimator does not require any prior information about the texture pdf. More recently, a large interest in this approach is observed in the radar literature. The M-estimator was rediscovered in [5], [18]. Pascal et al. proved the existence and uniqueness of its solution, the convergence of its recursive scheme under any initialization [7], and that it is asymptotically complex Wishart distributed [8]. Many applications to analysis of PolSAR data have followed, such as [9].

\section{Comparison and Discussion}

Four different approaches to covariance matrix estimation have been presented: the G-ML, K-ML and AK-ML estimators and the M-estimator. Table I gathers the key differences between the estimators. Note that all estimators except the G-ML must be solved iteratively. Due to different pdf assumptions of the texture in the product model, the primary difference between each estimator comes down to the weighting function $w(q)$. The input $q$ represents $\boldsymbol{s}_{k}^{\dagger}(\hat{\boldsymbol{C}})^{-1} \boldsymbol{s}_{k}$, whose values are scattered around its expectation value of $E\{q\}=d$.

In Fig. 1, the behavior of the weighting functions, $w_{G}, w_{K}$, $w_{A K}$ and $w_{M}$, are compared under various texture conditions, ranging in shape parameter $\alpha=1$ to 10 . Due to the capability to adapt to various texture conditions, the weighting function in the K-ML estimator is obviously more flexible compared to $w_{G}$ and $w_{M}$. This was also shown in [5]. We have found experimentally that the approximation error of $w_{A K}$ increases as $q$ and $\alpha$ go to zero, whereas the relative error is worst at $\alpha=d+1$. Still, the approximation performs well in general. However, the flexibility shown by the K-ML and AK-ML approaches requires the additional task of shape parameter estimation. This will introduce extra variance to the covariance matrix estimation. 
TABLE I

COVARIANCE MATRIX ESTIMATORS COMPARISON.

\begin{tabular}{|c|l|c|c|}
\hline & Weighting functions & Texture pdf assumption & Parameter estimation \\
\hline ML & $w_{o}(q)=h_{d+1}(q) / h_{d}(q)$ & no specific model & depend on model \\
\hline G-ML & $w_{G}(q)=1$ & constant variable & no need \\
\hline K-ML & $w_{K}(q)=\sqrt{\alpha / q} \cdot K_{\alpha-d-1}(\sqrt{4 \alpha q}) / K_{\alpha-d}(\sqrt{4 \alpha q})$ & gamma distribution & shape parameter \\
\hline & $\quad\left(\beta_{d}+\frac{2 q}{\tau_{0_{d}}} / \beta_{d+1}+\frac{2 q}{\tau_{0_{d}+1}}\right)^{\frac{1}{2}} \times \tau_{0_{d+1}}^{1+\beta_{d+1}} / \tau_{0_{d}}^{1+\beta_{d}}$ & & \\
AK-ML & $w_{A K}(q)=\quad$ gamma distribution & shape parameter \\
& $\quad \times \exp \left(\left(\frac{q}{\tau_{0_{d}}}-\frac{q}{\tau_{0_{d}+1}}\right)+\alpha\left(\tau_{0_{d}}-\tau_{0_{d+1}}\right)\right)$ & & no need \\
\hline $\mathbf{M}$ & $w_{M}(q)=d / q$ & no specific model & n \\
\hline
\end{tabular}

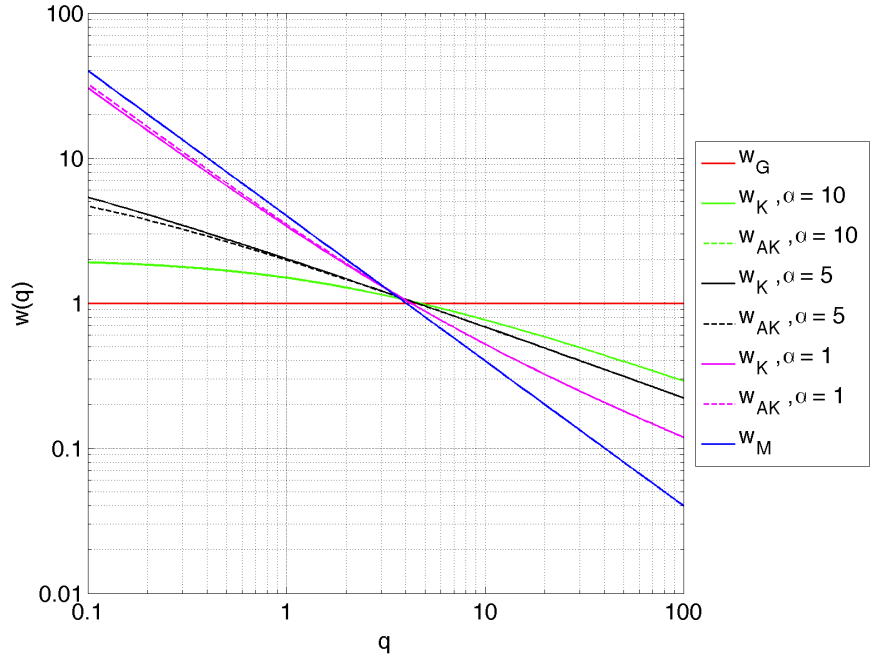

Fig. 1. The covariance matrix estimator weighting functions, $w_{G}, w_{K}, w_{A K}$ and $w_{M}$, at different specified shape parameters $\alpha$ in logarithmic scale.

\section{EXPERIMENTS AND RESULTS}

To evaluate the performance of the covariance matrix estimators discussed in Section III, a number of multivariate $\mathrm{K}$ distributed scattering vectors are simulated with a population mean covariance matrix obtained from real sea clutter in a quad-pol SAR dataset. In the experiments, various texture conditions are considered by specifying the shape parameters used in the simulation ( $\alpha=1$ to 20 ). The estimate from the G-ML estimator is used to initialize all the iterative estimators in this study. Monte Carlo simulations are implemented with sample sizes of 64, 256 and 1024. Note that a small number of samples is often preferred in practice, because of the increasing risk of non-stationary statistics in large estimation windows.

In this study, the Kullback-Leibler (KL) matrix distance derived under the Wishart model [19] is applied in the comparison of the estimation accuracy of the covariance matrix estimators. Its symmetric measure is defined as

$$
D_{K L}=\frac{\operatorname{tr}\left(\boldsymbol{C}^{-1} \hat{\boldsymbol{C}}\right)+\operatorname{tr}\left(\hat{\boldsymbol{C}}^{-1} \boldsymbol{C}\right)}{2}-d,
$$

where $\operatorname{tr}(\cdot)$ is the trace operator, $\hat{\boldsymbol{C}}$ and $\boldsymbol{C}$ represent the estimated and reference mean covariance matrix, respectively. We also compare the covariance matrix estimators with respect to computational efficiency, which is represented by the computation time and the number of iterations.

\section{A. Estimation accuracy}

Fig. 2 shows the experimental results of the covariance matrix estimation comparison. Note that a shape parameter estimate of $\hat{\alpha}>50$ can in practice be considered as a nontextured case, i.e., the sample of scattering vectors is complex multivariate Gaussian distributed.

From Fig. 2, it is clear that the K-ML estimator (black line) provides the best estimation results under all conditions, i.e. the smallest KL matrix distance. The new proposed AKML estimator (black dashed line) is very close to the exact K-ML, especially with a limited but practical number of samples. However, when a large sample size is available, it shows some relative performance loss under high texture conditions. The other two approaches, G-ML (red line) and M (blue line), are much less flexible. The G-ML estimator shows a large estimation error under high texture conditions. The M-estimator generally does not approach the accuracy of the ML estimators unless for extremely high texture cases. These results are explained by our theoretical study of the weighting functions in Section III-C, where the $w_{G}$ and $w_{M}$ cannot adapt to the range of texture conditions. An increase in estimation accuracy with larger sample sizes is seen as expected. However, the relative performance across different estimators stays consistent with little variations.

\section{B. Computational efficiency}

Table II shows a comparison of computation time and number of iterations for covariance matrix estimation under different texture conditions with 256 samples. In our experiments, convergence is defined as less than $0.001 \%$ change in the matrix determinant between iterations. The number of iterations required to reach convergence varies between several to dozens of times, and it increases with the texture level. A limit of 50 iterations is set to avoid an excessive number of iterations under extremely high texture conditions.

Obviously, the simple G-ML estimator, $\hat{\boldsymbol{C}}_{G}$, is the most efficient. It is followed by the M-estimator, $\hat{\boldsymbol{C}}_{M}$, which

TABLE II

MEAN COMPUTATION TIME [ $m s$ ] AND NUMBER OF ITERATIONS FOR COVARIANCE MATRIX ESTIMATION UNDER LOW ( $\alpha=11-20)$, MODERATE ( $\alpha=6$-10) AND HIGH $(\alpha=1-5)$ TEXTURE CONDITIONS WITH 256 SAMPLES AND 10,000 REPETITIONS

\begin{tabular}{|c|rrrr|}
\hline Textures & $\hat{\boldsymbol{C}}_{G}$ & $\hat{\boldsymbol{C}}_{K}$ & $\hat{\boldsymbol{C}}_{A K}$ & $\hat{\boldsymbol{C}}_{M}$ \\
\hline low & $0.194 / \mathbf{0}$ & $6.69 / \mathbf{4 . 4}$ & $4.27 / \mathbf{4 . 3}$ & $2.13 / \mathbf{3 . 6}$ \\
moderate & $0.174 / \mathbf{0}$ & $9.27 / \mathbf{6 . 8}$ & $5.60 / \mathbf{6 . 8}$ & $2.00 / \mathbf{3 . 7}$ \\
high & $0.136 / \mathbf{0}$ & $20.0 / \mathbf{1 8}$ & $12.7 / \mathbf{2 1}$ & $1.64 / \mathbf{3 . 9}$ \\
\hline
\end{tabular}




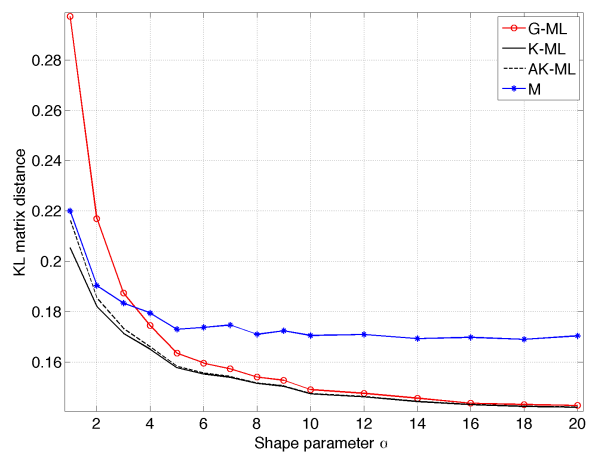

(a) Number of samples $=64$.

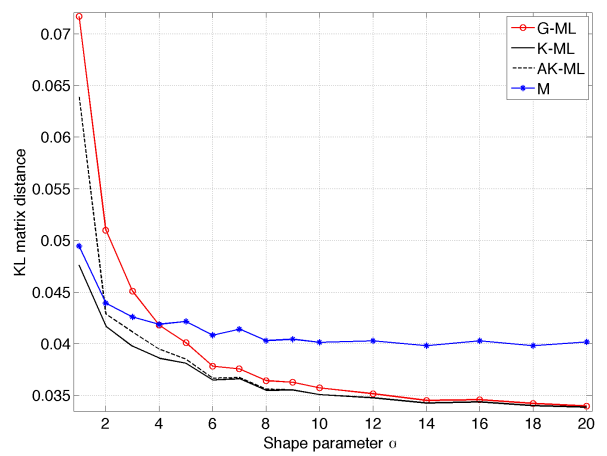

(b) Number of samples $=256$.

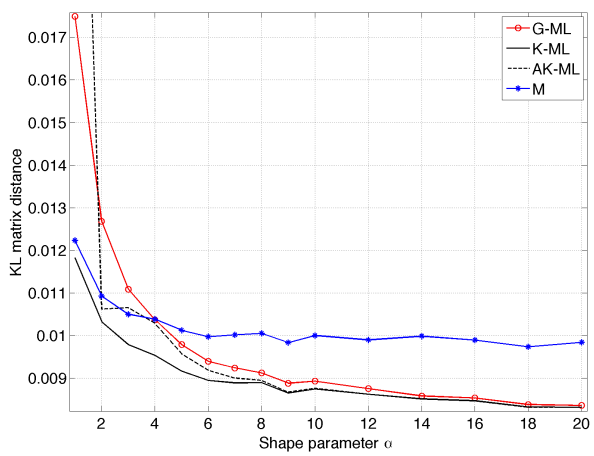

(c) Number of samples $=1024$.

Fig. 2. G-ML, K-ML, AK-ML and M covariance matrix estimation accuracy in terms of the KL matrix distance at specified shape parameters with 64 , 256 and 1024 samples. Each data point represents the mean value of 2000 repetitions.

also shows consistently short computational time due to its fast converging, uncomplicated, but non-adaptive weighting function. In general, the K-ML and AK-ML covariance matrix estimators, $\hat{\boldsymbol{C}}_{K}$ and $\hat{\boldsymbol{C}}_{A K}$, are more time consuming than the others, which is mainly because of their sophisticated weighting functions and the iterative computations. The AK-ML is still significantly faster than the K-ML, and the speed-up effect is most noticeable under high texture conditions. The savings also increase when the number of polarimetric channels, $d$, is lowered. This happens because the weighting function then take up a larger proportion of the estimator's computation time, since other operations such as matrix inversion and computation of the quadratic form $q$ become less costly. In total, the AK-ML estimator provides a compromise between accuracy and speed, which may make it more attractive for operational applications than what the K-ML has been.

\section{Conclusions}

A set of relevant approaches to covariance matrix estimation were investigated in the context of sea clutter statistical modeling via a comparative study of the G-ML, K-ML and AKML estimators, and the robust M-estimator. The estimation accuracy and the computational efficiency were measured to evaluate estimator performance, and need to be balanced according to user needs to make a choice.

In summary, the G-ML estimator is fast due to its simplicity, and it is expected to perform well under low to moderate texture conditions. The K-ML estimator models the sea clutter more accurately than the G-ML estimator under high texture, but at increased computational cost. The newly proposed AKML estimator is comparable to the K-ML, especially with small sample sizes, when only a small loss in accuracy is observed under high texture conditions. Its computational cost is significantly lower than for the K-ML estimator under all conditions. The M-estimator does not provide any distinct advantages in any of the studied cases. However, because it requires no assumption of the texture distribution, it makes an alternative to the G-ML in highly textured clutter.

\section{REFERENCES}

[1] F. Gini and J. Michels, "Performance analysis of two covariance matrix estimators in compound-Gaussian clutter," IEE Proc. Radar, Sonar and Navigation, vol. 146, no. 3, pp. 133-140, Jun. 1999.
[2] P. W. Vachon and J. Wolfe, "Validation of ship signatures in Envisat ASAR AP mode data using AISLive: Data acquisition, processing, and analysis results." Defence Research and Development Canada, Ottawa, Canada, Tech. Rep., Mar. 2008.

[3] D. Tao, S. N. Anfinsen, and C. Brekke, "Ocean clutter modeling for ship detection," in Proc. SEASAR 2012, Troms $\varnothing$, Norway, Jun. 2012, p. 6.

[4] L. Novak and M. Burl, "Optimal speckle reduction in polarimetric SAR imagery," IEEE Trans. Aerosp. Electron. Syst., vol. 26, no. 2, pp. 293 305, Mar. 1990.

[5] F. Gini and M. Greco, "Covariance matrix estimation for CFAR detection in correlated heavy tailed clutter," Signal Processing, vol. 82, no. 12, pp. $1847-1859$, Dec. 2002.

[6] D. E. Tyler, "A distribution-free M-estimator of multivariate scatter," Ann. Statist., vol. 15, no. 1, pp. 234-251, Mar. 1987.

[7] F. Pascal, Y. Chitour, J.-P. Ovarlez, P. Forster, and P. Larzabal, "Covariance structure maximum-likelihood estimates in compound Gaussian noise: Existence and algorithm analysis," IEEE Trans. Signal Process., vol. 56, no. 1, pp. 34-48, 2008.

[8] F. Pascal, P. Forster, J.-P. Ovarlez, and P. Larzabal, "Performance analysis of covariance matrix estimates in impulsive noise," IEEE Trans. Signal Process., vol. 56, no. 6, pp. 2206-2217, Jun. 2008.

[9] G. Vasile, J.-P. Ovarlez, F. Pascal, and C. Tison, "Coherency matrix estimation of heterogeneous clutter in high-resolution polarimetric SAR images," IEEE Trans. Geosci. Remote Sens., vol. 48, no. 4, pp. 18091826, Apr. 2010.

[10] S. H. Yueh, J. A. Kong, J. K. Jao, R. T. Shin, and L. M. Novak, "Kdistribution and polarimetric terrain radar clutter," J. Electrom. Waves Applic., vol. 3, no. 8, pp. 747-768, 1989.

[11] A. Farina, F. Gini, M. Greco, and L. Verrazzani, "High resolution sea clutter data: statistical analysis of recorded live data," IEE Proc. Radar, Sonar and Navigation, vol. 144, no. 3, pp. 121-130, Jun. 1997.

[12] D. Iskander and A. Zoubir, "Estimation of the parameters of the Kdistribution using higher order and fractional moments," IEEE Trans. Aerosp. Electron. Syst., vol. 35, no. 4, pp. 1453-1457, Oct. 1999.

[13] S. N. Anfinsen, "On the supremacy of logging," in Proc. POLinSAR 2011, Frascati, Italy, Jan. 2011, p. 8.

[14] C. Oliver and S. Quegan, Understanding Synthetic Aperture Radar Images, ser. SciTech radar and defense series. SciTech Publ., 2004.

[15] N. Goodman, "Statistical analysis based on a certain multivariate complex Gaussian distribution (an introduction)," Ann. Math. Stat., vol. 34 no. 1, pp. 152-177, Mar. 1963.

[16] K. Sangston, F. Gini, M. Greco, and A. Farina, "Structures for radar detection in compound Gaussian clutter," IEEE Trans. Aerosp. Electron. Syst., vol. 35, no. 2, pp. 445-458, Apr. 1999.

[17] C. G. Small, Expansions and Asymptotics for Statistics. Boca Raton, USA: CRC Press, 2010.

[18] E. Conte, A. De Maio, and G. Ricci, "Covariance matrix estimation for adaptive CFAR detection in compound-Gaussian clutter," IEEE Trans. Aerosp. Electron. Syst., vol. 38, no. 2, pp. 415-426, Apr. 2002.

[19] A. Frery, A. Nascimento, and R. Cintra, "Analytic expressions for stochastic distances between relaxed complex Wishart distributions," IEEE Trans. Geosci. Remote Sens., 2013 (IEEE Early Access). 